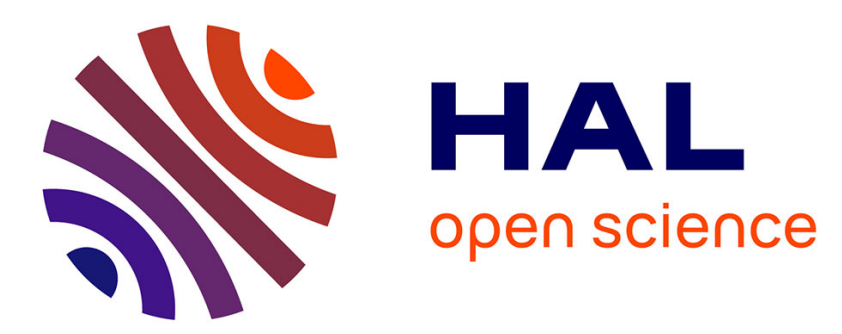

\title{
Analysis of Heart Rate Variability Using Time-Varying Filtering of Heart Transplanted Patients
}

\author{
Ghailen Laouini, Olivier Meste, Marianna Meo
}

\section{To cite this version:}

Ghailen Laouini, Olivier Meste, Marianna Meo. Analysis of Heart Rate Variability Using TimeVarying Filtering of Heart Transplanted Patients. International Conference of the IEEE Engineering in Medicine and Biology Society, Aug 2012, United States. hal-00738441

\section{HAL Id: hal-00738441 \\ https://hal.science/hal-00738441}

Submitted on 4 Oct 2012

HAL is a multi-disciplinary open access archive for the deposit and dissemination of scientific research documents, whether they are published or not. The documents may come from teaching and research institutions in France or abroad, or from public or private research centers.
L'archive ouverte pluridisciplinaire HAL, est destinée au dépôt et à la diffusion de documents scientifiques de niveau recherche, publiés ou non, émanant des établissements d'enseignement et de recherche français ou étrangers, des laboratoires publics ou privés. 


\title{
Analysis of Heart Rate Variability Using Time-Varying Filtering of Heart Transplanted Patients
}

\author{
Ghailen Laouini, Olivier Meste and Marianna Meo, member IEEE
}

\begin{abstract}
In this paper, we analyze the heart rate variability (HRV), obtained by using the time-varying integral pulse frequency modulation (TVIPFM) which is well adapted to the exercise stress testing.

We consider that the mean heart period is varying function of time, during exercise. This technique allows the estimation of the autonomic nervous system modulation (ANS) from the beat occurrences. The estimated respiratory sinus arrhythmia is then filtered in the time-frequency domain around the respiration using a time-varying filter. It is proven that the Spectrogram is a convenient time-frequency representation that allows the implementation of such filter. The recorded data comes from exercise test performed by ten heart transplant patients. The magnitude of the filtered modulation of the heart rate due to respiration is compared to the date of transplantation taking into account the volume of respiration. It reveals that the normalized magnitude of the filtered variability, is significantly increased as the age of transplantation is higher with a high correlation coefficient $(R=0.74, p=0.01)$. This correlation raised to 0.82 when considering dynamic behavior of the parameters.

Applied to our dataset, standard parameter fails to exhibit such correlation.
\end{abstract}

\section{INTRODUCTION}

Several works [1] have used the Integral pulse frequency modulation (IPFM) model to explain the regulation of the heart rate by the ANS, in different physiological situations, even during stress testing exercise [9]. However the IPFM model assumes a constant threshold [2,3] considering a constant mean heart period. During dynamic protocol the use of the time-varying threshold IPFM (TVIPFM) model to correct the heart rate variability is relevant for the estimation of the modulating signal $[4,10]$.

Many authors have emphasized the close relationship between respiration and HRV $[6,7,8]$. This component appears to be the principal non stationary modulation of high frequency. Since it is embedded in a mixture of modulating signals, its analysis requires a filtering that is time-varying in that case. The performance of stress test exercises allows the use of the respiratory sinus arrhythmia (RSA) information for the ANS tone assessment. This modulation is effective with respects to the innervation of the heart. After orthotopic heart transplantation the loss of the innervation should suppress this modulation. During long-term follow-up it has been shown [11] that the HRV is higher as long is the age of the transplantation, with a strong correlation. In the study [11] the variability measurement is addressed by using the standard deviation of the data during resting conditions.

G. Laouini is with Laboratory I3S, University of Nice and CNRS, Sophia Antipolis 06903, France, (e-mail:laouini@i3s.unice.fr)

O. Meste is with Laboratory I3S, University of Nice and CNRS, Sophia Antipolis 06903, France, (e-mail:meste@i3s.unice.fr)
Thus a correlation between this parameter and the age of the transplantation is exhibited under controlled protocol, limiting the impact of such analysis. It would be of greater interest to show this correlation with relaxed and dynamic conditions corresponding to increasing physical exercise.

The paper is organized as follows. The methods and materials are presented in Section II, where we use a mathematical development to demonstrate the feasibility of filtering in the time-frequency domain, using the Spectrogram. The TVIPFM model is described as well in this section to obtain a corrected HRV signal. Section III presents the results and Section IV the discussion.

\section{METHODS}

\section{A. Mathematical development}

In this study the signal to be filtered is non stationary. It means that the characteristics of the bandpass filter must vary with time. The time-frequency transformation that will permit this particular filtering is the Spectrogram defined by:

$$
S(t, f)=\left|\int x(s) h^{*}(s-t) e^{-i 2 \pi f s} d s\right|^{2}
$$

where $h(t)$ stands for the smoothing window. In the following it will be demonstrated that by using this representation in addition with a matched template $G(t, f)$, an efficient time-varying filter can be applied. Note that continuous time is considered in the entire development but results are still valid with sampled time. The spectrogram being the squared magnitude of the short Fourier Transform, it is linked to the Wigner-Ville (WV) distribution by the convolution products in time and frequency:

$$
S(t, f)=\left(W_{h} * * W_{x}\right)(t, f)
$$

Assuming that the WV transform of $h(t)$ is separable such that $W_{h}(t, f)=g(t) l(f)$ (verified when $h(t)$ is a gaussian function), when $x(t)$ is a linear frequency modulation, i.e. $x(t)=\exp \left(i \pi \alpha t^{2}\right)$, the Spectrogram is then:

$$
\begin{gathered}
S(t, f)=\iint g(t-s) l(f-\nu) \delta(s-\alpha \nu) d s d \nu \\
S(t, f)=\int g(t-\alpha \nu) l(f-\nu) d \nu
\end{gathered}
$$

Note that the signal $x(t)$ is locally approximated by this model whose modulation slope is $\alpha$. This assumption is valid with respect to the respiration component where even during exercise the frequency varies smoothly. This quantity 
is nonzero when the two windows $g$ and $l$ are simultaneously nonzero.

$$
\left\{\begin{array}{lll}
g(t) \neq 0 & \text { for } & -T<t<T \\
l(f) \neq 0 & \text { for } & -F<f<F
\end{array}\right.
$$

where $g(t)$ and $l(f)$ are assumed hermitian symmetric.Then:

$$
\left\{\begin{array}{l}
g(t-\alpha \nu) \neq 0 \quad \text { for } \quad \frac{-T+t}{\alpha}<\nu<\frac{+T+t}{\alpha} \\
l(f-\nu) \neq 0 \text { for } \quad-\stackrel{F}{F}+\nu<f<F+\nu
\end{array}\right.
$$

It can be concluded that for a given time $t_{0}$

$$
-F+\frac{-T+t_{0}}{\alpha}<\nu<F+\frac{+T+t_{0}}{\alpha}
$$

So $\left[-F+\frac{-T+t_{0}}{\alpha} ; F+\frac{+T+t_{0}}{\alpha}\right]$ is the support of $S\left(t_{0}, f\right)$ along the frequency axis. The mean value of this interval is $\frac{t_{0}}{\alpha}$ that corresponds to the instantaneous frequency of $x(t)$ for $t=t_{0}$. As shown in the sequel, because this result means that the support is centered on the instantaneous frequency of $x(t)$, the integration around this interval will provide the magnitude without bias. This allows us to impose to the template $G(t, f)$, that will be multiplied with $S(t, f)$, to be nonzero around $f=\frac{t_{0}}{\alpha}$ for $t=t_{0}$ in order to get rid off the other components. It is well known that for the Spectrogram, the marginal is:

$$
\int S(t, f) d f=\left(p_{x} * p_{h}\right)(t)
$$

with $p_{x}(t)$ being the squared envelop $|x(t)|^{2}$ of $x(t)$. If this envelop is locally a constant $A$, then the computation of this integration will produce $A^{2}$ if $\int|h(t)|^{2}=1$. This result imposes to the smoothing window $h(t)$ to be energy normalized. The slow variation of the respiration component in the heart period perfectly match the local assumption.

This demonstration proves the role of the time-varying filtering around the respiration to retrieve the envelope of the respiration component observed within the HRV signal. This modulation will be corrected or not by the TVIPFM model.

The template $G(t, f)$ that conveys the knowledge of the respiration frequency is defined as:

$$
\left\{\begin{array}{l}
G(t, f)=1 \text { if } \quad f \in B_{R} \quad \forall t \\
G(t, f)=0 \quad \text { otherwise }
\end{array}\right.
$$

With $B_{R}$ is the band frequency of respiration. Details on the computation of the time-varying filter using $G(t, f)$ is fully provided in [7].

\section{B. The time-varying threshold Integral pulse frequency modulation model}

The TVIPFM model is based on the hypothesis that the ANS influence on the sinus atrial SA node can be represented by the modulating signal $m(t)$.

In the TVIPFM model the integral of $1+m(t)$ is compared to a time-varying threshold $T(t)$, representing the time-varying mean heart period. Assuming that the first beat occurs at time $t_{0}=0$, the beat occurrence time series can be generated as solution of [5]:

$$
k=\int_{0}^{t_{k}} \frac{1+m(t)}{T(t)} d t
$$

with its extension to continuous case:

$$
k(t)=\int_{0}^{t} \frac{1+m(s)}{T(s)} d s
$$

the instantaneous heart rate is defined as below

$$
d_{H R}(t)=\frac{1+m(t)}{T(t)}=\frac{d k(t)}{d t}
$$

Supposing the variations of the term $\frac{1}{T(t)}$ are slower than those of the term $\frac{m(t)}{T}$, and their spectral components don't overlap, we define the time-varying heart rate as:

$$
d_{H R M}(t)=\frac{1}{T(t)}
$$

and the heart rate variability signal is defined as

$$
d_{H R V}(t)=d_{H R}(t)-d_{H R M}(t)=\frac{m(t)}{T(t)}
$$

So, to obtain the modulating signal, we have to correct the HRV signal $d_{H R V}(t)$ by the time-varying mean heart rate $d_{H R M}(t)$.

In the TVIPFM, the variations of the time-varying threshold $T_{A C}(t)$, are small compared to its mean value $T_{D C}$. Rewriting the instantaneous heart rate

$$
\begin{gathered}
d_{H R}(t)=\frac{1+m(t)}{T(t)}=\frac{1+m(t)}{T_{D C}+T_{A C}(t)}=\frac{1+m(t)}{T_{D C}\left(1+\frac{T_{A C}(t)}{T_{D C}}\right)} \\
\approx \frac{1+m(t)}{T_{D C}}\left(1+\frac{T_{A C}(t)}{T_{D C}}\right)
\end{gathered}
$$

with the assumption $m(t)<1$, we can neglect the second order term, then we obtain:

$$
d_{H R}(t)=\frac{1+m(t)-\frac{T_{A C}(t)}{T_{D C}}}{T_{D C}}
$$

we assume

$$
d_{H R M}(t)=\frac{1-\frac{T_{A C}(t)}{T_{D C}}}{T_{D C}}=\frac{1}{T_{D C}}
$$

To obtain the modulating signal $m(t)$, first we estimate $d_{H R}(t)$, where $k(t)$ is estimated from pairs $\left(t_{k}, k\right)$, then we estimate the $d_{H R M}(t)$ term by low-pass filtering $d_{H R}(t)$ and we calculate the $d_{H R V}(t)$ term. Finally, the modulating signal is obtained as:

$$
m(t)=\frac{d_{H R V}(t)}{d_{H R M}(t)}
$$




\section{MATERIALS AND RESULTS}

Recorded signals have been obtained from surface electrodes that produce interferences such as noise and baseline wander. So, to exploit all signals we must cancel the disturbances caused by breathing. First we filter the signal using 500-th order high-pass finite impulse response filter in order to remove the baseline generated by the respiration. After using a threshold technique we demodulate the filtered signal to detect all the time occurrences of R-waves, namely the $t_{k}$. The differences of consecutive $t_{k}$ provide the heart period. Finally, the HRV is computed by removing the trend of the heart period. In addition to the ECG, the respiration signal is recorded to extract the frequency and the volume. Ten heart transplant subjects with different ages of transplantation [4160] months are analyzed by using different parameters. The parameter introduced in [11] is indeed a standard deviation (stand) evaluated on the HRV at rest. It will be compared to the quantity $\operatorname{mag}(t)$ corresponding to the magnitude of the time-varying filtered respiration component extracted from the HRV. When corrected by the TVIPFM model the subscript will be $t v$. Because the respiration volume has not been controlled during the protocol, the time-varying quantities will be normalized by the respiration volume computed from the respiration signal. This normalization denoted by the subscript resp will be exclusively applied to mag because it contains only the respiration influence. All these quantities are computed at rest and at the maximum of the exercise. An interval of 100 seconds will be used to compute parameter stand and to average the quantities $\operatorname{mag}(t)$. In summary, the analysis will provide the parameters stand, mag, mag $_{t v}$ and mag $_{t v, r e s p}$.

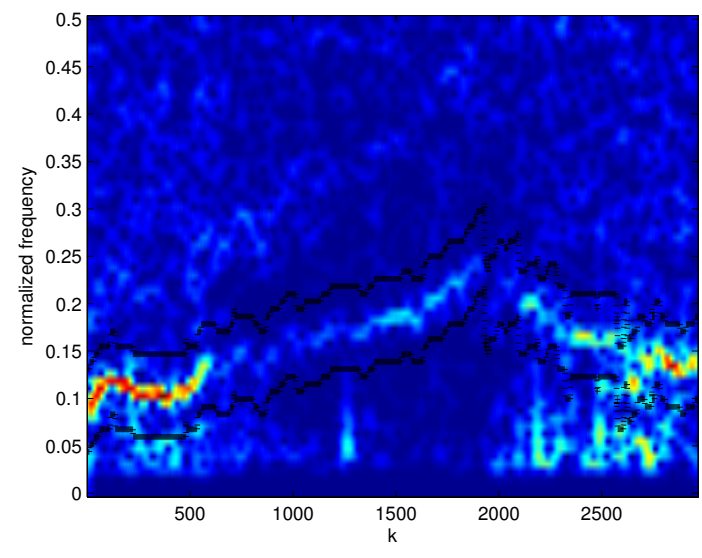

Fig. 1. The spectrogram of the heart rate variability with the template $G(t, f)$ boundaries (in black) defined by the respiratory frequency band

Figure (1), reveals that the RSA modulation is high at the beginning of exercise, then the magnitude of this modulation decreases abruptly after the beginning of exercise due to the exercise pressor reflex causing ventilatory responses to exercise. Then period from second 1400 to 1800 corresponds to the increasing exercise. The intensity of the RSA is at lower values during this interval. During the recovery, there is an abrupt increase which gradually decreases as the heart rate decreases reaching values close to those observed at the beginning of the test.
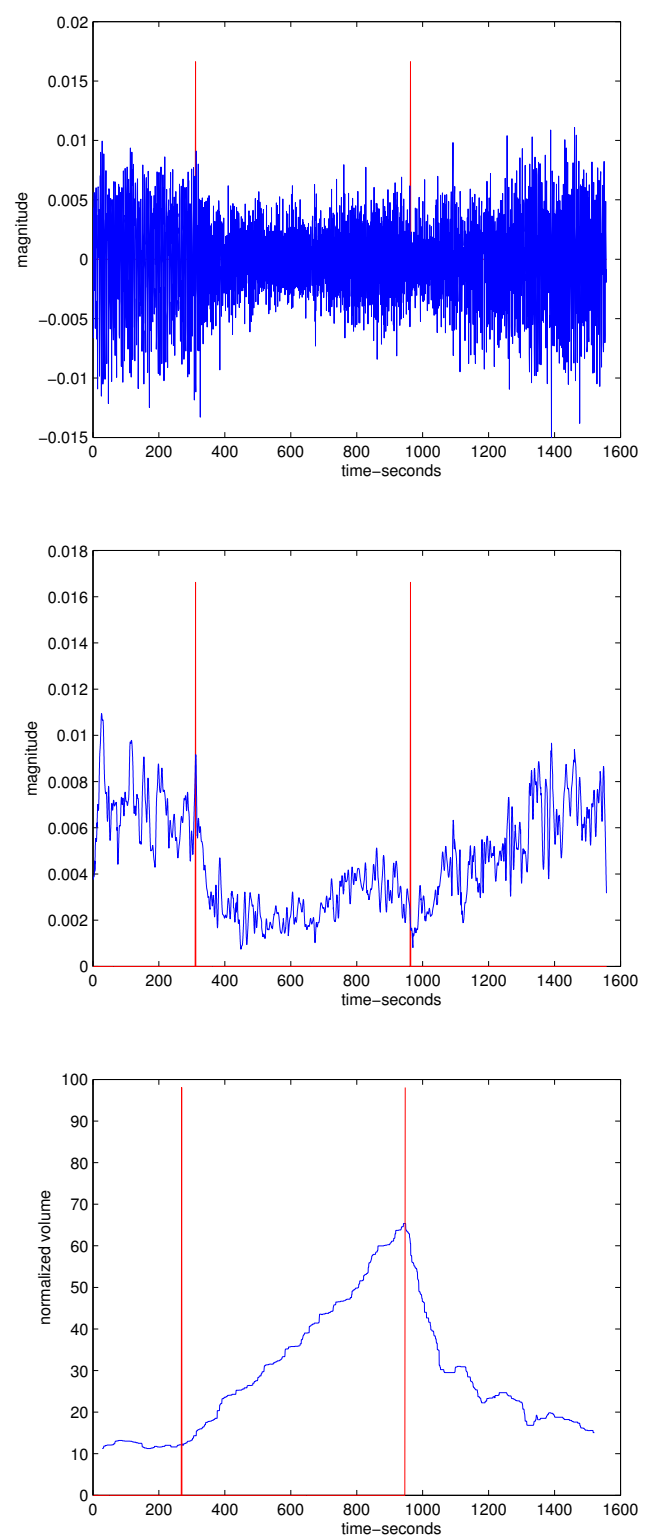

Fig. 2. The corrected variability with the TVIPFM model (top), the magnitude of the envelop filtered around the respiration frequency (middle) and the breathing volume (bottom), the red vertical lines correspond to the begining and the maximum of the exercise, respectively. Note that the magnitude unit is meaningless because of the correction procedure, the volume has been normalized by the greater value within all the subjects

Figure (2) shows first the HRV corrected with the TVIPFM model (upper). This variability is then filtered in the timefrequency domain, producing an estimation of the RSA magnitude (middle). If needed, the volume of the respiration is computed from the respiration signal (bottom). From this function of time (middle) an average over 100 seconds is computed in the resting period and at the maximum of the exercise. 
TABLE I

THE CORRELATION OF PARAMETERS WITH THE AGE OF TRANSPLANTATION

\begin{tabular}{|c||c|c|c|}
\hline & $($ rest $)$ & $($ max$)$ & $($ rest)-(max) \\
\hline stand & $\mathrm{R}=0.25, \mathrm{p}=0.48$ & $\mathrm{R}=0.27, \mathrm{p}=0.45$ & $\mathrm{R}=0.11, \mathrm{p}=0.76$ \\
\hline mag & $\mathrm{R}=0.43, \mathrm{p}=0.22$ & $\mathrm{R}=-0.21, \mathrm{p}=0.56$ & $\mathrm{R}=0.29, \mathrm{p}=0.40$ \\
\hline mag $_{\text {tv }}$ & $\mathrm{R}=0.61, \mathrm{p}=0.06$ & $\mathrm{R}=-0.14, \mathrm{p}=0.69$ & $\mathrm{R}=0.67, \mathrm{p}=0.03$ \\
\hline mag $_{\text {tv }}$ resp & $\mathrm{R}=0.74, \mathrm{p}=0.01$ & $\mathrm{R}=0.09, \mathrm{p}=0.80$ & $\mathrm{R}=0.82, \mathrm{p}=0.003$ \\
\hline
\end{tabular}

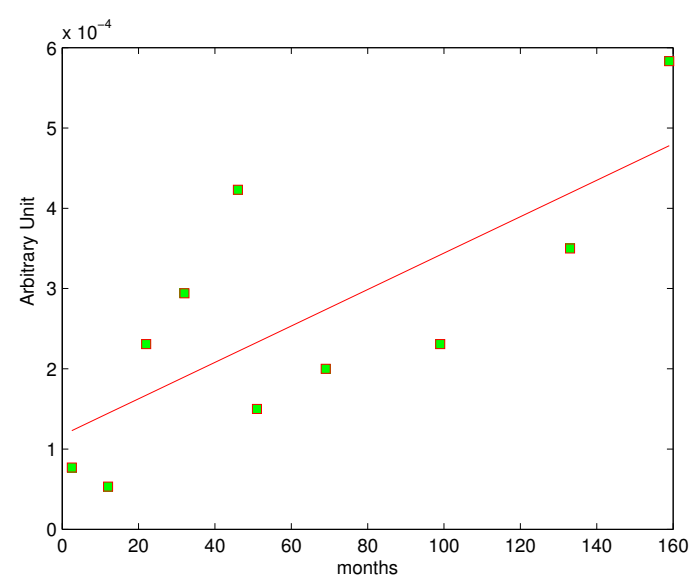

Fig. 3. At rest. The magnitude of the heart rate variability corrected with TVIPFM model filtered around the frequency band of respiration and normalized with the respiratory volume, function of the age of transplantations

Table I, displays all the parameters extracted from the ten patients. Its clear that mag $_{t v, r e s p}$ outperforms the other parameters whereas the stand parameter fails in exhibiting a linear correlation. The difference (rest)-(max) is provided in order to investigate a dynamic behavior. Once again, mag $_{t v, \text { resp }}$ shows a clear correlation. Figure (3), represents the magnitude of the heart rate variability $\operatorname{mag}_{t v, r e s p}($ rest), filtered around the frequency band of respiration and normalized with the respiratory volume, function of the age of transplantation. It shows the high correlation $(\mathrm{R}=0.74$, $\mathrm{P}=0.01$ ), between the age of the transplantation and the RSA with TVIPFM correction and respiration normalization.

One of the important results in this paper is that from the parameters obtained from the HRV, only the variability corrected by the TVIPFM and normalized is highly correlated with the age of the transplantation. The result was expected from the literature but with a parameter that fails in that case (stand). However, since a higher variability is related to a reinnervation, our result is in line with the physiology knowledge.

\section{Discussion}

In this paper, a method of time-varying filtering is proposed, centering the filter around the respiration frequency band. The relevance of using the Spectrogram for this aim is proven. In combination with this filter we use the TVIPFM model which has a time-varying mean heart period adapted to non stationary conditions. The time-varying mean heart rate is estimated by low-pass filtering $d_{H R}(t)$. Thus, the modulating signal is filtered in the time-frequency domain and the instantaneous power is then calculated by integrating over frequencies to obtain the magtv $_{t v}$.

Probably because no respiration profiles have been imposed in the protocol, the normalization with the respiration volume has increased the correlation. In contrast to standard parameter, namely the standard deviation stand, the proposed one corroborates the physiologic expectation on our data set. The reinnervation should be higher as the age of the transplantation is longer, inducing a higher variability. Note that the global variability addressed by the parameter stand didn't convey any positive result. The high correlation between the RSA and the age of transplantation is due to the TVIPFM correction and the time-varying filtering. This significant correlation is revealed at rest and not during exercise. The dynamic behavior corresponding to (rest)(max) is even more correlated. This result is of great interest because it highlights the gain of the HRV dynamics due to the innervation process.

\section{REFERENCES}

[1] E. Bayly, Spectral analysis of pulse frequency modulation in the nervous system. IEEE Trans Biom., vol. BME-15, pp. 257-265, 1968.

[2] J. Mateo and P. Laguna, Improved hart rate variability time-domain signal construction from the beat occurence time according to IPFM model. IEEE Trans Biom., vol. 47, pp. 985-996, 2000.

[3] J. Mateo and P. Laguna, Analysis of heart rate variability in the presence of ectopic beats using the heart timing signal. IEEE Trans Biom., vol. 50, no. 3, pp. 334-343, 2003.

[4] R. Bailon, G. Laouini, M. Orini, P. Laguna, O. Meste, The integral pulse frequency modulation model with time-varying threshold: application to heart rate variability analysis during exercise stress testing. IEEE Trans Biom., vol. 58, no. 3, pp. 642-652, 2011.

[5] O. Rompleman, J. Snijders and C. van Spronsen, The measurement of heart rate variability spectra with the help of a personal computer. IEEE Trans Biom., vol. 29, no. 7, pp. 503-510, 1982.

[6] O. Meste, G. Blain and S. Bermon. Some considerations on the IPFM Model for the heart rate variability analysis.Computers in Cardiology., 2003, p. 709-712, 2003.

[7] O. Meste, B. Khaddoumi, G. Blain and S. Bermon. Time-varying analysis methods and models for the respiratory and cardiac system coupling in graded exercise. IEEE Transactions on Biomedical Engineering., vol. 52, no. 11, pp. 1921-1930, 2005.

[8] G. Blain G, O. Meste and S. Bermon. Assessment of ventilatory thresholds during graded and maximal exercise test using time-varying analysis of respiratory sinus arrythmia. Br. J. Sports Med., vol 39, no. 7, pp. 448-452, 2005.

[9] R. Bailon G, L. Mainardi, M. Orini, L. Sornmo, and P. Laguna. Analysis of heart rate variability during exercise stress testing using respiratory information, Biomed Signal Process Control., vol. 5, no. 4, pp. 299-310, 2010.

[10] G. Laouini, A. Cabasson, G. Blain, P. Bonizzi, O. Meste, and S. Bermon, Evidence of the influence of respiration on the heart rate variability after human heart transplantation: Role of observation model, in Proc. Comput. Cardiol., vol. 36, pp. 409-412, 2009. http://cinc.mit.edu, 2009

[11] A. F. Folino, G. Buja, M. Miorelli, U. Livi, A. Nava, G. Thiene, S. Dalla Volta, Heart rate variability in patients with orthotopic heart transplantation: Long-term follow-up., Clinical cardiology, vol. 16, no. 7, pp. 539-542, 1993 